\title{
Numerically solving generated Jacobian equations in freeform optical design
}

\author{
Lotte B. Romijn ${ }^{1, *}$, Martijn J.H. Anthonissen ${ }^{1, * *}$, Jan H.M. ten Thije Boonkkamp ${ }^{1, * * *}$, and Wilbert L. IJzerman ${ }^{1,2, * * * *}$ \\ ${ }^{1}$ Eindhoven University of Technology (TU/e), Department of Mathematics and Computer Science, PO Box 513, 5600 MB Eindhoven, \\ The Netherlands \\ ${ }^{2}$ Signify Research, High Tech Campus 7, 5656 AE Eindhoven, The Netherlands
}

\begin{abstract}
We present an efficient numerical algorithm that can be used to solve the generalized Monge-Ampère equations for a single freeform reflector and lens surface. These equations are instances of so-called 'generated Jacobian equations' which are characterized by associated generating functions. The algorithm has a wide applicability to any optical system that can be described by a smooth generating function.
\end{abstract}

\section{Introduction}

We consider two optical systems: (System 1) a lens surface for a point source and far-field target intensity, and (System 2) a reflector surface for a parallel incoming beam and near-field target illuminance. For System 1 the source emits light radially outward and we consider a source emittance in spherical coordinates $f_{1}(\phi, \theta)$ in $[1 \mathrm{~m} / \mathrm{sr}]$. The target intensity distribution $g_{1}(\psi, \chi)$ in $[1 \mathrm{~m} / \mathrm{sr}]$ in the far field is expressed with respect to a different set of spherical coordinates $(\psi, \chi)$ with origin the lens surface approximated as a point in space (far-field approximation). The first surface of the lens is spherical and the second surface is freeform. For System 2 we are given an emittance of the source domain $f_{2}(\mathrm{x})$ in $\left[1 \mathrm{~m} / \mathrm{m}^{2}\right]$ and a target illuminance in the near field given by $g_{2}(\mathrm{y})$ in $\left[\mathrm{lm} / \mathrm{m}^{2}\right]$, where $\mathrm{x}$ and $\mathrm{y}$ are the local Cartesian coordinates of the source and target planes, respectively. In figure 1 a schematic representation is given for System 2. Our goal is to compute the optical surfaces that define the mappings $\boldsymbol{m}_{1}$ and $\boldsymbol{m}_{2}$ transforming $f_{1}$ into $g_{1}$ and $f_{2}$ into $g_{2}$, respectively.

\section{Mathematical model}

In previous work on System 1 [1], we used optimal transport theory to derive a relation of the form $u_{1}(\boldsymbol{x})+u_{2}(\boldsymbol{y})=$ $c(\boldsymbol{x}, \boldsymbol{y})$, where $u_{1}$ specifies the location of the freeform optical surface and $c(\boldsymbol{x}, \boldsymbol{y})$ is a logarithmic cost function in optimal transport theory with $(\boldsymbol{x}, \boldsymbol{y})$ denoting the stereographic coordinates obtained from performing coordinate transformations on the source and target domains. Subsequently, we could derive an optical map $\boldsymbol{m}$ and derive the generalized Monge-Ampère equation as

$$
\operatorname{det}(\operatorname{Dm})=\frac{\operatorname{det}(\boldsymbol{P}(\boldsymbol{x}))}{\operatorname{det}(\boldsymbol{C}(\boldsymbol{x}, \boldsymbol{m}(\boldsymbol{x})))}=\frac{f_{1}(\boldsymbol{x})\left(1+|\boldsymbol{m}(\boldsymbol{x})|^{2}\right)^{2}}{g_{1}(\boldsymbol{m}(\boldsymbol{x}))\left(1+|\boldsymbol{x}|^{2}\right)^{2}},
$$

\footnotetext{
*e-mail: 1.b.romijn@tue.nl

**e-mail: m.j.h.anthonissen@tue.nl

***e-mail: j.h.m.tenthijeboonkkamp@tue.nl

****e-mail: wilbert.ijzerman@signify.com
}

where D $\boldsymbol{m}$ is the Jacobi matrix of $\boldsymbol{m}, \boldsymbol{P}$ is a symmetric positive-definite (SPD) matrix and $\boldsymbol{C}=\mathrm{D}_{x y} c=\left(c_{x_{i} y_{j}}\right)$ is the matrix of mixed second-order partial derivatives with respect to the stereographic coordinates of the source and target.

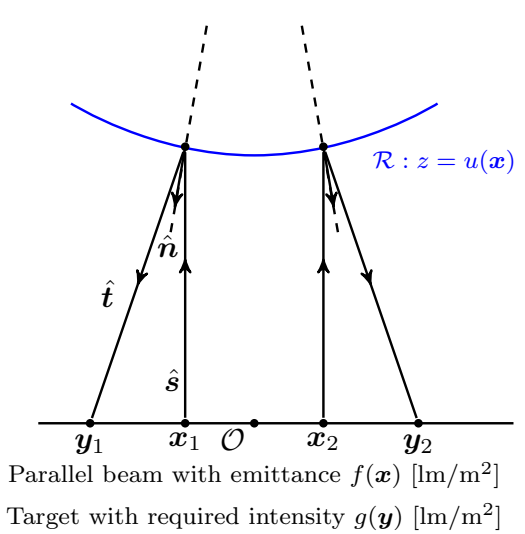

Figure 1: Schematic representation of a parallel beam of light rays, reflected by the reflector surface onto a target in the near field.

However, for some optical systems such as System 2 a relation of the form $u_{1}(\boldsymbol{x})+u_{2}(\boldsymbol{y})=c(\boldsymbol{x}, \boldsymbol{y})$ does not exist in any coordinate system. In this paper, we generalize the cost-function formulation in optimal transport theory to a generating-function formulation by defining $u(\boldsymbol{x})=G(\boldsymbol{x}, \boldsymbol{y}, z(\boldsymbol{y}))$ and $z(\boldsymbol{y})=H(\boldsymbol{x}, \boldsymbol{y}, u(\boldsymbol{x}))$, where $u$ is the unknown location of the optical surface, $G$ and $H$ are smooth functions and $G(\boldsymbol{x}, \boldsymbol{y}, \cdot)$ and $H(\boldsymbol{x}, \boldsymbol{y}, \cdot)$ are each other's inverses. For both systems, we can reformulate the generalized Monge-Ampère equation as a generated Jaco- 

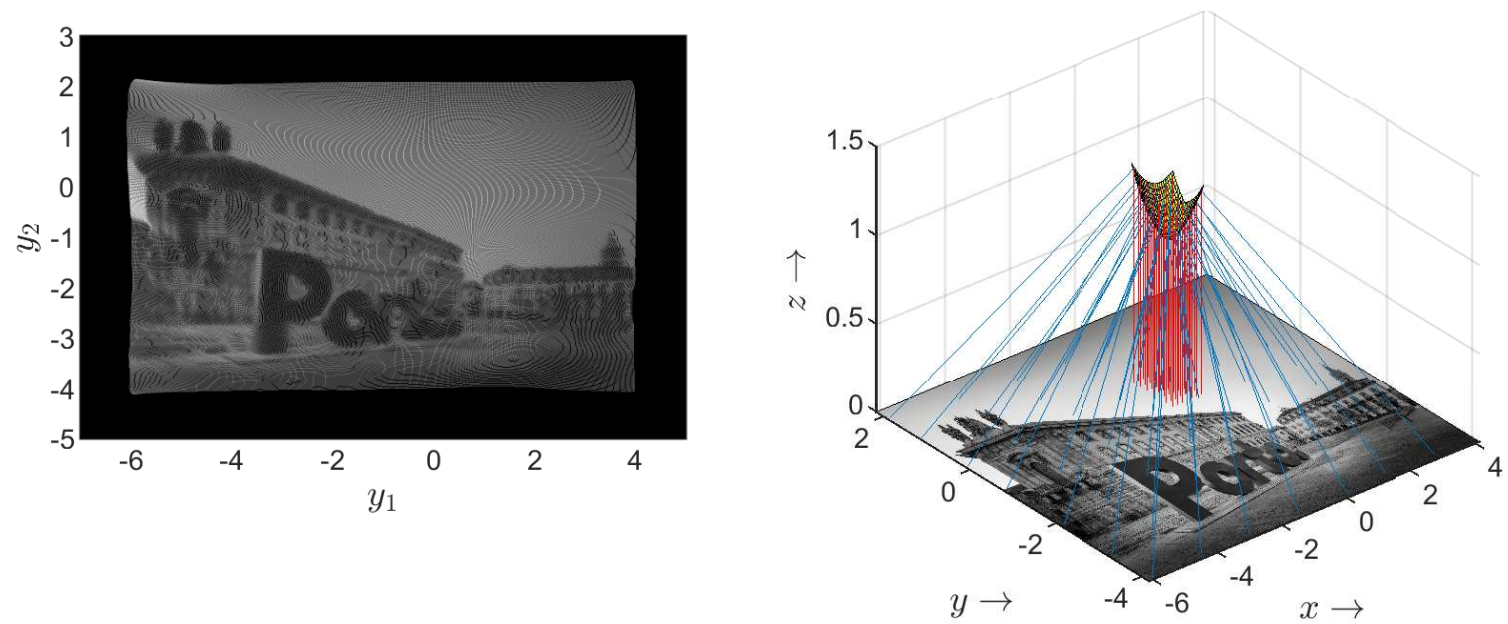

Figure 2: (Left) Ray traced image of Porto projected on a screen in the near field. (Right) Cartoon of a single freeform reflector that converts a small parallel beam (in red) into an output beam (in blue) that produces a picture of Porto on a screen in the near field. Only a small subset of the total rays traced (approximately 100.000) has been plotted.

bian equation [2]

$$
\operatorname{det}(\mathrm{Dm})=\frac{\operatorname{det}(\boldsymbol{P}(\boldsymbol{x}))}{\operatorname{det}(\boldsymbol{C}(\boldsymbol{x}, \boldsymbol{m}(\boldsymbol{x}), u(\boldsymbol{x})))}=\frac{F(\boldsymbol{x})}{G(\boldsymbol{m}(\boldsymbol{x}))},
$$

where $\boldsymbol{P}$ is a symmetric positive-definite (SPD) matrix and we redefine $\boldsymbol{C}$ as $\boldsymbol{C}=\mathrm{D}_{x y} H(\boldsymbol{x}, \boldsymbol{m}(\boldsymbol{x}), u(\boldsymbol{x}))=\left(H_{x_{i} y_{j}}\right)$. Note the added dependency of $\boldsymbol{C}$ on the surface $u$. For System 1, we consider the stereographic coordinates $(\boldsymbol{x}, \boldsymbol{y})$ and have $F(\boldsymbol{x})=f_{1}(\boldsymbol{x}) /\left(1+|\boldsymbol{x}|^{2}\right)^{2}$ and $G(\boldsymbol{m}(\boldsymbol{x}))=g_{1}(\boldsymbol{m}(\boldsymbol{x})) /(1+$ $\left.|\boldsymbol{m}(\boldsymbol{x})|^{2}\right)^{2}$. For System 2, we take Cartesian coordinates $\boldsymbol{x}=\mathrm{x}$ and $\boldsymbol{y}=\mathrm{y}$, and derive $F(\boldsymbol{x})=f_{2}(\mathrm{x})$ and $G(\boldsymbol{m}(\boldsymbol{x}))=$ $g_{2}(\boldsymbol{m}(\mathrm{x}))$.

For System 1, we use Hamilton's angular characteristic and derive $H(\boldsymbol{x}, \boldsymbol{y}, u(x))=u(\boldsymbol{x})\left(n-1+\frac{2|\boldsymbol{x}-\boldsymbol{y}|^{2}}{\left(1+|\boldsymbol{x}|^{2}\right)\left(1+|\boldsymbol{y}|^{2}\right)}\right)$. Inverting this equation gives the generating function

$$
G(\boldsymbol{x}, \boldsymbol{y}, z(\boldsymbol{y}))=z(\boldsymbol{y})\left(n-1+\frac{2|\boldsymbol{x}-\boldsymbol{y}|^{2}}{\left(1+|\boldsymbol{x}|^{2}\right)\left(1+|\boldsymbol{y}|^{2}\right)}\right)^{-1} .
$$

For System 2, the function $H$ is Hamilton's point characteristic $H(\boldsymbol{x}, \boldsymbol{y}, u(\boldsymbol{x}))=u(\boldsymbol{x})+\sqrt{|\boldsymbol{y}-\boldsymbol{x}|^{2}+u(\boldsymbol{x})^{2}}$ which is equal to the optical path length from a point $x$ on the source domain to a point $\boldsymbol{y}$ on the target domain, see figure 1. Inverting this equation gives the generating function $G(\boldsymbol{x}, \boldsymbol{y}, z(\boldsymbol{y}))=u$ with $z(\boldsymbol{y})=1 / H$ as derived in $[2,3]$

$$
G(\boldsymbol{x}, \boldsymbol{y}, z)=\frac{1}{2 z}-\frac{z}{2}|\boldsymbol{x}-\boldsymbol{y}|^{2} .
$$

\section{Numerical method}

Using a generalized least-squares approach [4] we iteratively update the mapping $\boldsymbol{m}$. We write the generalized Monge-Ampère equation as the matrix equation $\boldsymbol{C D m}(\boldsymbol{x})=\boldsymbol{P}(\boldsymbol{x})$, where $\boldsymbol{P}(\boldsymbol{x})$ satisfies $\operatorname{det}(\boldsymbol{P}(\boldsymbol{x}))=$
$F(\boldsymbol{x}) / G(\boldsymbol{m}(\boldsymbol{x}))$, cf. [5]. An iterative procedure is used to find the mapping, which involves an efficient procedure to find the numerical solution of a constrained minimization problem for each grid point to compute $\boldsymbol{P}$ and the solution of a linear elliptic boundary value problem to compute $\boldsymbol{m}$. In order to update the matrix $\boldsymbol{C}$ during the iterative procedure, the location of the optical surface is calculated from the mapping also in a least-squares sense.

The numerical method can be used to compute reflector and lens surfaces for various examples. For instance, we can compute the reflector surface that transforms light of a parallel beam into a near-field picture of Porto, as shown in figure 2. For System 1 we will compare the results of the cost-function approach previously presented in [1] to the new generating-function approach.

By using a generating-function approach we are able to extend the applicability of the least-squares procedure originally presented in [5] to any optical system that can be described by a smooth generating function.

\section{References}

[1] L. Romijn, J. ten Thije Boonkkamp, W. IJzerman, JOSA A 36, 1926 (2019)

[2] N.S. Trudinger, Discrete Contin. Dyn. Syst. 34, 1663 (2012)

[3] F. Jiang, N. Trudinger, N.S. Arch. Ration. Mech. Anal 229, 547 (2018)

[4] L.B. Romijn, J.H. ten Thije Boonkkamp, W.L. IJzerman, J. Comput. Phys. 408, 109283 (2020)

[5] C. Prins, Ph.D. thesis, Eindhoven University of Technology, Eindhoven (2014) 